\title{
The efficacy of infiltration
} anaesthesia for adult mandibular incisors: a randomised doubleblind cross-over trial comparing articaine and lidocaine buccal and buccal plus lingual infiltrations

\author{
A. Jaber, ${ }_{1}$ J. M. Whitworth, ${ }_{1}^{2}$ I. P. Corbett, ${ }^{3}$ B. Al-Baqshi, ${ }^{4}$ M. D. Kanaa ${ }^{5}$ \\ and J. G. Meechan ${ }^{6}$
}

Aim To compare the efficacy of 2\% lidocaine and 4\% articaine both with 1:100,000 adrenaline in anaesthetising the pulps of mandibular incisors. Methods Thirty-one healthy adult volunteers received the following local anaesthetic regimens adjacent to a mandibular central incisor: 1) buccal infiltration of $1.8 \mathrm{~mL}$ lidocaine plus dummy lingual injection $(L B), 2)$ buccal plus lingual infiltrations of $0.9 \mathrm{~mL}$ lidocaine $(L B L), 3)$ buccal infiltration of $1.8 \mathrm{~mL}$ articaine plus dummy lingual injection $(A B), 4)$ buccal plus lingual infiltrations of $0.9 \mathrm{~mL}$ articaine (ABL). Pulp sensitivities of the central incisor and contralateral lateral incisor were assessed electronically. Anaesthetic efficacy was determined by two methods: 1) Recording the number of episodes with no responses to maximal electronic pulp tester stimulation during the course of the study period, 2) recording the number of volunteers with no response to maximal pulp tester stimulation within 15 min and maintained for $45 \mathrm{~min}$ (defined as sustained anaesthesia). Data were analysed by McNemar, chi-square, MannWhitney and paired t-tests. Results For both test teeth, the number of episodes of no sensation on maximal stimulation was significantly greater after articaine than lidocaine for both techniques. The split buccal plus lingual dose was more effective than the buccal injection alone for both solutions ( $p<0.001)$. $4 \%$ articaine was more effective than $2 \%$ lidocaine when comparing sustained anaesthesia in both teeth for each technique $(p<0.001)$, however, there was no difference in sustained anaesthesia between techniques for either tooth or solution. Conclusions $4 \%$ articaine was more effective than 2\% lidocaine (both with 1:100,000 adrenaline) in anaesthetising the pulps of lower incisor teeth after buccal or buccal plus lingual infiltrations.

\section{INTRODUCTION}

Effective pulpal anaesthesia is essential for many dental procedures, and predictable local anaesthetic regimens are important in reducing the fear and anxiety that dentistry might provoke. Despite a range of commercially available local anaesthetic drugs, lidocaine remains the most commonly employed in dentistry in the United Kingdom, with an excellent safety

\footnotetext{
PhD student, ${ }^{2}$ Clinical Consultant/Senior Lecturer, ${ }^{3}$ Clinical Lecturer, Oral and Maxillofacial Surgery, ${ }^{4}$ Specialist Registrar, Endodontics, ${ }^{5} \mathrm{PhD}$ student, ${ }^{6 *}$ Senior Lecturer, Oral and Maxillofacial Surgery, School of Dental Sciences, Newcastle University, Newcastleupon-Tyne, NE2 4BW

*Correspondence to: Dr John Meechan Email: j.g.meechan@ncl.ac.uk
}

\section{Online article number E16}

Refereed Paper - accepted 6 August 2010

DOI: 10.1038/sj.bdj.2010.974

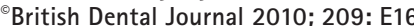

record. ${ }^{1}$ Recent studies ${ }^{2-4}$ have shown that 4\% articaine with adrenaline, which was first marketed in the United Kingdom in 1999 , is more effective than $2 \%$ lidocaine with adrenaline for mandibular permanent first molar anaesthesia following buccal infiltration in the lower jaw.

Regional blocks do not always result in successful pulpal anaesthesia for lower anterior teeth. ${ }^{5,6}$ Meechan and Ledvinka ${ }^{7}$ investigated infiltration anaesthesia in the adult mandibular incisor region and found that the combination of buccal and lingual infiltrations was more effective than the use of either in isolation. Their study included only lidocaine with adrenaline. Nuzum et al. ${ }^{8}$ have recently suggested that supplementing a buccal infiltration of 4\% articaine with 1:100,000 adrenaline with a lingual injection of the same solution increases the efficacy of pulpal anaesthesia for lower lateral incisors.

The purpose of the current study was to compare the efficacy of 2\% lidocaine and 4\% articaine both with 1:100,000 adrenaline in anaesthetising the pulps of ipsilateral mandibular central incisors and contralateral mandibular lateral incisors (to determine spread of anaesthesia) after buccal infiltration or a split buccal plus lingual dose. The primary outcome measure was pulp anaesthesia (negative response to electronic pulp testing). The secondary outcomes were onset time and injection discomfort.

The null hypothesis tested was that there are no significant differences between $4 \%$ articaine and 2\% lidocaine infiltrations in the degree of pulpal anaesthesia and onset of pulpal anaesthesia in 
ipsilateral central incisors and contralateral lateral incisors after infiltrations in the anterior mandible.

\section{MATERIALS AND METHODS}

Approval for this prospective, randomised, double-blind, cross-over study was secured from the national ethics committee and from the UK Medicines and Healthcare products Regulatory Agency. A formal power calculation based on the data from earlier investigations ${ }^{2,7}$ indicated that a sample size of 31 volunteers would provide a 90\% chance of finding a difference at the 0.05 level. Healthy adult volunteers 18 years old and over were included in this study. Exclusion criteria included:

1. under 18 years of age

2. unable to give informed consent

3. bleeding disorders

4. facial anaesthesia or paraesthesia

5. allergies to local anaesthetic drugs

6. pregnant at the time of the study

7. teeth that responded negatively to baseline pulp testing or with key test teeth missing.

Each subject had at least one vital permanent mandibular central and contralateral lateral incisor. Clinical examinations were performed to ensure that all test teeth were free of caries, large restorations, and periodontal disease, and that none had a history of trauma or sensitivity.

The following local anaesthetic regimens were applied in randomised order determined by a web-based program (http:// department.obg.cuhk.edu.hk/researchsupport/random_integer.asp) at the right permanent mandibular central incisor over four visits, at least one week apart:

1. $1.8 \mathrm{~mL}$ of $2 \%$ lidocaine with

1:100,000 adrenaline (Dentsply

Pharmaceutical, Konstanz, Germany)

as a buccal infiltration in the muc-

cobuccal fold with a dummy injection (needle penetration only) lingually in the lingual reflected mucosa (LB)

2. $0.9 \mathrm{~mL}$ of $2 \%$ lidocaine with

$1: 100,000$ adrenaline as a buccal infiltration in the muccobuccal fold and $0.9 \mathrm{~mL}$ as a lingual infiltration in the lingual reflected mucosa (LBL)

3. $1.8 \mathrm{~mL}$ of $4 \%$ articaine with 1:100,000 adrenaline (Septodont, Saint-Maur-des-Fosses, France) as a buccal infiltration in the muccobuccal fold with a dummy injection lingually $(\mathrm{AB})$

4. $0.9 \mathrm{~mL}$ of $4 \%$ articaine with $1: 100,000$ adrenaline as a buccal infiltration in the muccobuccal fold and $0.9 \mathrm{~mL}$ as a lingual infiltration in the lingual reflected mucosa (ABL).

All injections were administered by the same investigator, using a standard aspirating dental cartridge syringe (Ultra Safety plus XL Syringe, Septodont) fitted with a 30-gauge dental needle. This investigator had no participation in measuring outcome. Injections were administered at a rate of 15 seconds per $0.9 \mathrm{~mL}$. The dummy injections involved needle penetration for 15 seconds without deposition of local anaesthetic solution. Dummy injections were administered to blind the volunteers to the method of anaesthesia used.

The efficacy of anaesthesia was determined by electronic pulp testing (Analytic Technology, Redmond, WA, USA) by an investigator blinded to the injections administered. The pulp tester was set to deliver a 0 -80 digital reading on a rate setting of five, corresponding to a non-linear increasing voltage, zero to maximum, over 30 seconds. Calibration of the pulp tester demonstrated a maximum voltage of 270 volts at an output impedance of $140 \mathrm{~K}$ Ohms.

Testing was performed on the appropriate mandibular central incisor and the contralateral mandibular lateral incisor twice before injection, at two-minute intervals after injection until 30 minutes and then at five-minute intervals until 45 minutes postinjection. The timings were measured by stopwatch. An unanaesthetised maxillary central incisor was tested before injection and at 10 and 45 minutes post-injection to ensure proper function of the pulp tester.

Anaesthetic efficacy was determined by two methods:

1. The number of episodes of no response to maximal pulp tester stimulation (80 reading) during the post-injection trial period

2. The number of volunteers with no response to maximum pulp tester stimulation (80 reading) within $15 \mathrm{~min}$ and maintained for $45 \mathrm{~min}$ post-injection after each treatment (sustained anaesthesia).
The onset of pulpal anaesthesia was considered as the first of two or more episodes of no sensation to maximal stimulation (80 reading).

Subjective discomfort associated with each of the injections and dummy injections was also recorded by volunteers on $100 \mathrm{~mm}$ visual analogue scales with end-points marked 'No pain' (0 mm) and 'Unbearable pain' (100 mm).

Data were analysed in SPSS (SPSS 17.0, SPSS Inc., Chicago, IL) by McNemar, chisquare, Mann-Whitney, and Student's paired t-test. The Bonferroni correction was applied where multiple comparisons were made.

\section{RESULTS}

Thirty-one volunteers completed the investigation (11 male, 20 female; mean age $24.4 \mathrm{yrs}, \mathrm{SD}=4.4 \mathrm{yrs}$ ).

Figures 1 and 2 show the percentage of volunteers reporting negative response to maximal pulp stimulation (80 reading) at time intervals after injection in ipsilateral mandibular central incisors and contralateral mandibular lateral incisors.

For ipsilateral central incisors, the number of episodes of no response to maximal electronic pulp stimulation (80 reading) was significantly different among the four anaesthetic regimens (chisquare $=326.6, p<0.001)$. The number of episodes of no sensation in ipsilateral central incisors was greater after AB (453 episodes) than LB (244). This difference was significant (McNemar test, $p<0.01$ ). A significant difference was also found between ABL (499 episodes) and LBL (348 episodes) [McNemar test, $\mathrm{p}<0.01$ ]. ABL resulted in significantly more episodes of no sensation than $\mathrm{AB}$ alone (499 versus 453 respectively, McNemar test $\mathrm{p}<0.01)$. LBL also resulted in significantly more episodes of no sensation than LB alone (348 versus 244 respectively, McNemar test $\mathrm{p}<0.01)$.

For contralateral lateral incisors, there were again highly significant differences in the number of episodes of negative pulp testing among the four anaesthetic regimens (chi-square $=241.2, \mathrm{p}<0.001$ ). For the contralateral lateral incisor, the incidence of maximal stimulation without sensation was significantly higher after $A B$ than LB (234 versus 88 episodes respectively, McNemar test $\mathrm{p}<0.01)$. Significant differences were also found between $\mathrm{ABL}$ 


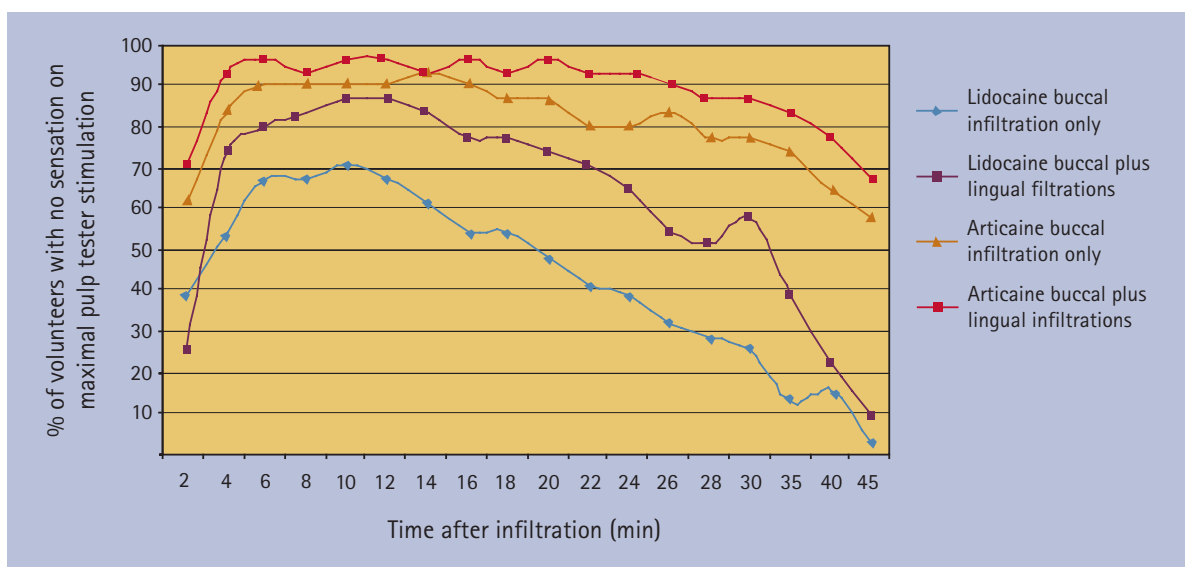

Fig. 1 Percentage of volunteers reporting negative response to maximal pulp stimulation (80 reading) at time intervals in ipsilateral mandibular central incisors after articaine and lidocaine buccal and buccal plus lingual infiltrations

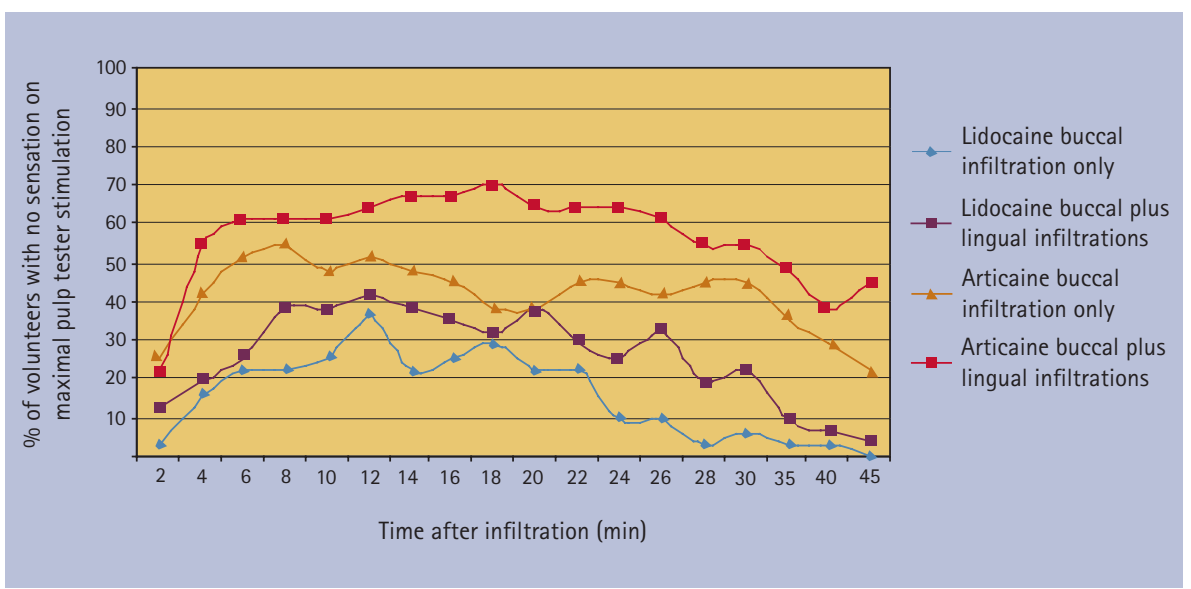

Fig. 2 Percentage of volunteers reporting negative response to maximal pulp stimulation ( 80 reading) at time intervals in contralateral mandibular lateral incisors after articaine and lidocaine buccal and buccal plus lingual infiltrations

Table 1 Anaesthetic success (sustained anaesthesia) in ipsilateral central incisors and contralateral lateral incisors after each infiltration method

\begin{tabular}{|c|c|c|c|c|c|c|c|c|c|}
\hline & \multicolumn{2}{|l|}{ LB } & \multicolumn{2}{|c|}{ LBL } & \multicolumn{2}{|l|}{$A B$} & \multicolumn{2}{|c|}{$A B L$} & \multirow{3}{*}{$\begin{array}{l}P \\
\text { value }\end{array}$} \\
\hline & \multicolumn{2}{|c|}{ Success } & \multicolumn{2}{|c|}{ Success } & \multicolumn{2}{|c|}{ Success } & \multicolumn{2}{|c|}{ Success } & \\
\hline & $\mathrm{N}$ & $\%$ & $\mathrm{~N}$ & $\%$ & $\mathrm{~N}$ & $\%$ & $\mathrm{~N}$ & $\%$ & \\
\hline Ipsilateral central incisors & 1 & 3.2 & 3 & 9.7 & 14 & 45.2 & 20 & 64.5 & 0.001 \\
\hline Contralateral lateral incisors & 0 & 0 & 1 & 3.2 & 7 & 22.6 & 12 & 38.7 & 0.001 \\
\hline
\end{tabular}

and LBL (319 versus 146 episodes respectively, McNemar test, $\mathrm{p}<0.01)$. The difference between LB alone and LBL (88 episodes versus 146 episodes) was also significant [McNemar test, $\mathrm{p}<0.01$ ]. A similar difference was also found for $\mathrm{AB}$ alone (234 episodes) compared with ABL (319 episodes) [McNemar test, $\mathrm{p}<0.01$ ].

When sustained anaesthesia was compared, the following results were found (Table 1). For ipsilateral central incisors, there were highly significant between LB alone (one volunteer [3.2\%]) and LBL (three volunteers [9.7\%]) [McNemar test, $\mathrm{p}=0.5$ ]. Similarity, there was no significant difference between $A B$ alone (14 volunteers [45.2\%]) and ABL (20 volunteers [64.5\%]) [McNemar test, $\mathrm{p}=0.146]$.

For contralateral lateral incisors, there were again significant differences between the four anaesthetic regimens (chisquare $=22.4, \mathrm{p}<0.001)$. For contralateral lateral incisors, none of the volunteers (0\%) achieved sustained anaesthesia following LB compared to seven (22.6\%) after AB. The difference was significant (McNemar test, $\mathrm{p}<0.01)$. Twelve volunteers $(38.7 \%)$ secured sustained pulp anaesthesia following ABL compared to one (3.2\%) after LBL. This difference was significant (McNemar test, $\mathrm{p}<0.01)$. No significant difference was found between LB alone (no volunteers) and LBL (one volunteer) Again, there was no significant difference between $A B$ alone and ABL (seven volunteers [22.6\%] versus 12 volunteers [38.7\%] respectively [McNemar test, $\mathrm{p}=0.125]$ ).

Non-parametric testing was employed to assess differences in the onset of pulpal anaesthesia since the data were not normally distributed. Teeth that did not achieve no response to the maximum stimulus from the pulp tester on at least two consecutive occasions were not included in the onset data analysis. Table 2 shows the data for the onset of anaesthesia in ipsilateral central incisors and contralateral lateral incisors. There was no difference in the median time of onset of pulpal anaesthesia in the ipsilateral central incisor after $\mathrm{LB}$ and $\mathrm{AB}$ (2.0 min for both). The median time of onset of pulp anaesthesia was shorter after ABL (2.0 min) than LBL (4.0) (Mann-Whitney test, $\mathrm{p}<0.01$ ). A significant difference was found between the median time of onset of pulp anaesthesia after LB and LBL (2.0 min versus $4.0 \mathrm{~min}$ respectively, Mann-Whitney test, $\mathrm{p}<0.05$ ). There was no significant difference in the median time of onset of pulp anaesthesia after $\mathrm{AB}$ compared to $\mathrm{ABL}$ (2.0 min for both).

For the contralateral lateral incisor, there was no significant difference in the median time of onset of pulp anaesthesia after $\mathrm{LB}$ and $\mathrm{AB}$ (5.0 versus 4.0 respectively; Mann-Whitney test, $\mathrm{p}=0.097$ ). The median onset time was shorter after 
Table 2 Onset of pulpal anaesthesia in minutes in ipsilateral central incisors and contralateral lateral incisors after each infiltration method

\begin{tabular}{l|l|l|l|l|l|l|l|l|}
\hline & \multicolumn{3}{|c|}{ Ipsilateral central incisors } & \multicolumn{3}{c}{ Contralateral lateral incisors } \\
\cline { 2 - 11 } & LB & LBL & AB & ABL & LB & LBL & AB & ABL \\
\hline Mean & 3.4 & 3.8 & 3.3 & 2.3 & 6.8 & 5.0 & 5.5 & 5.0 \\
\hline Median & 2.0 & 4.0 & 2.0 & 2.0 & 5.0 & 6.0 & 4.0 & 4.0 \\
\hline Range & $2-6$ & $2-8$ & $2-14$ & $2-4$ & $2-18$ & $2-10$ & $2-28$ & $2-14$ \\
\hline
\end{tabular}

LB: lidocaine buccal infiltration, LBL: lidocaine buccal and lingual infiltration, AB: articaine buccal infiltration, ABL: articaine and lingual infiltration

Table 3 VAS scores $(\mathrm{mm})$ of discomfort after real and dummy infiltrations

\begin{tabular}{|c|c|c|c|c|c|c|c|}
\hline & $\begin{array}{l}\text { LB } \\
(1.8 \mathrm{~mL})\end{array}$ & $\begin{array}{l}\text { LB } \\
(0.9 \mathrm{~mL})\end{array}$ & $\begin{array}{l}\mathrm{LL} \\
(0.9 \mathrm{~mL})\end{array}$ & $\begin{array}{l}\text { Lingual } \\
\text { penetration }\end{array}$ & $\begin{array}{l}A B \\
(1.8 \mathrm{~mL})\end{array}$ & $\begin{array}{l}A B \\
(0.9 \mathrm{~mL})\end{array}$ & $\begin{array}{l}\mathrm{AL} \\
(0.9 \mathrm{~mL})\end{array}$ \\
\hline Number & 31 & 31 & 31 & 31 & 31 & 31 & 31 \\
\hline Mean $(\mathrm{mm})$ & 32.9 & 34.7 & 23.3 & 12.5 & 36.8 & 33.5 & 24.9 \\
\hline $\mathrm{SD}(\mathrm{mm})$ & 19.1 & 22.0 & 17.2 & 13.9 & 22.8 & 21.4 & 20.9 \\
\hline Minimum (mm) & 3.0 & 2.0 & 2.0 & 1.0 & 2.0 & 1.0 & 1.0 \\
\hline Maximum (mm) & 70.0 & 76.0 & 69.0 & 53.0 & 81.0 & 78.0 & 68.0 \\
\hline
\end{tabular}

ABL (4.0 min) than LBL (6.0 min) (MannWhitney test, $\mathrm{p}<0.01)$. There was no significant difference in the median time of onset of pulp anaesthesia between LB and LBL (5.0 min versus 6.0 min respectively; Mann-Whitney test, $\mathrm{p}=0.435$ ). Similarly, there was no significant difference in the median time of onset of pulp anaesthesia between $\mathrm{AB}$ and $\mathrm{ABL}$ (4.0 min for both).

A summary of the VAS scores for injection discomfort is shown in Table 3. No significant differences were noted between the drugs and methods of administration. Lingual penetration was, however, more comfortable than lingual infiltration (Student's paired t-test $\mathrm{p}<0.01$ ).

\section{DISCUSSION}

The use of electronic pulp testing is well established in clinical practice and in local anaesthetic trials. ${ }^{9-12}$ A number of studies have used no response to maximal electronic pulp tester output (80 reading) as a measure of pulpal anaesthesia. ${ }^{13-20}$ Pulp anaesthesia can be defined in many ways, and with varying degrees of stringency. One method is to determine the number or percentage of subjects who obtain two or more consecutive episodes of maximal electronic pulp tester stimulation (80 reading) without response.,21-23 We employed a more stringent definition in this study, which was similar to those described by others. ${ }^{24,25}$

An impression of anaesthetic response can also be obtained by counting the number of negative responses to pulp testing across a study period, or by assessing shifts from baseline pulp testing. ${ }^{4}$ In patient studies, the 'acid test' of pulp anaesthesia is the ability to complete treatment without pain. This is clearly not possible in healthy volunteer studies, but it is generally assumed that negative pulp tester responses form a satisfactory proxy marker for pulp anaesthesia.

The results of the present study confirm those of Meechan and Ledvinka, ${ }^{7}$ Nuzum et $a l^{8}$ and Yonchak et al. ${ }^{21}$ that infiltrations can provide successful pulpal anaesthesia in mandibular anterior teeth. In the Meechan and Ledvinka study, ${ }^{7}$ a split buccal and lingual dose of lidocaine was more effective than a buccal infiltration alone on the basis of the number of no responses to maximal pulp tester stimulation across the study period. This improvement cannot be the result of an increase in local anaesthetic dose as an identical volume was given for both injection methods. A possible explanation for improved anaesthetic efficacy of the combination method is that the lingual infiltration might counter accessory innervation to the pulps of central incisors from the lingual and mylohyoid nerves.

The results of the present investigation, when using a similar analysis to that of Meechan and Ledvinka, ${ }^{7}$ support the findings of the latter workers. The split buccal and lingual technique was more effective than a buccal dose alone for both lidocaine and articaine. As far as drug efficacy is concerned, articaine was more effective than lidocaine. Articaine buccal infiltration produced more episodes of no response to maximum stimulation than lidocaine buccally in both ipsilateral central and contralateral lateral incisors, and the split articaine technique produced more episodes than the equivalent method with lidocaine.

When considering sustained anaesthesia, lidocaine buccal infiltration produced a very low success rate of $3.2 \%$ in the ipsilateral central incisor. This is lower than the $50 \%$ success reported by Meechan and Ledvinka ${ }^{7}$ following lidocaine buccal infiltration, but the definition of anaesthesia employed in their report was less stringent, requiring only a single episode of negative pulp testing within the trial period.

For both ipsilateral central and contralateral lateral incisors there were highly significant differences when sustained anaesthesia was compared between the four treatments, $(p<0.001)$. For both test teeth the results were significantly better when articaine was used irrespective of the technique employed. This is similar to the findings of others ${ }^{2-4}$ who reported that $4 \%$ articaine with $1: 100,000$ adrenaline is more effective than $2 \%$ lidocaine with 1:100,000 adrenaline when used for infiltration anaesthesia in the mandibular molar region.

The success of sustained anaesthesia in the present study for the ipsilateral central incisor after articaine was infiltrated both buccally and lingually (64.5\%) was comparable to the $63 \%$ reported by Yonchak and others ${ }^{21}$ after labial infiltrations of lidocaine with adrenaline, however these workers used a less stringent criterion for success. The present results are also similar to those obtained by Haas and others ${ }^{26}$ after buccal infiltrations of $1.5 \mathrm{~mL}$ of $4 \%$ articaine or $4 \%$ prilocaine both with 1:200,000 adrenaline adjacent to the mandibular canine tooth. In that study, anaesthetic success was 65\% for articaine and 50\% for prilocaine. Their 
definition of success was, however, less stringent, being no response from subjects to single episodes of maximal stimulation (80 reading). Nuzum et al. ${ }^{8}$ reported $98 \%$ anaesthetic success for the lateral incisor when a $1.8 \mathrm{~mL}$ buccal infiltration of $4 \%$ articaine with $1: 100,000$ adrenaline was supplemented with an identical injection lingually. This greater success compared to the present study is probably the result of the much higher dose (3.6 $\mathrm{mL})$ and also the fact that the definition of success (no response on two or more consecutive occasions to the maximum stimulation from the pulp tester) was less rigid in that study.

When considering sustained anaesthesia, anaesthetic success did not differ significantly between lidocaine buccal infiltration alone and when split buccally and lingually for both ipsilateral central and contralateral lateral incisors. Similar findings were true for articaine, with the buccal and split dose techniques securing similar levels of long-lasting pulp anaesthesia in both ipsilateral central and contralateral lateral incisors.

It is not possible from the results of the present study to determine if the superiority of the articaine solution over the lidocaine preparation is a feature of the higher concentration of the former or as a result of a greater inherent ability to diffuse.

It is clear from the current results that definitions of anaesthetic success can have an important bearing on reported outcomes. No standard has been defined within the local anaesthetic research community. Agreement of a standardised definition of anaesthetic success in volunteers would be an advance in this regard. Despite this lack of uniformity some of the results of this study (see Figure 1) suggest that the split buccal plus lingual technique with 4\% articaine with 1:100,000 adrenaline appears to be the method of choice for lower central incisor anaesthesia. This is in agreement with the findings of Nuzum et $a l .{ }^{8}$ The anaesthetic efficacy for the lateral incisor shown here suggests that infiltration at the contralateral central is probably insufficient for clinical use on those teeth. The data for the contralateral lateral incisor presented here support the view that 4\% articaine with adrenaline has a greater ability to diffuse that $2 \%$ lidocaine with adrenaline.
Several factors can affect the onset time of local anaesthesia including the site of administration, the method of administration and the intrinsic properties and concentration of the drug. The present results showed that the median onset time of pulpal anaesthesia for articaine (2.0 min) was significantly shorter than for lidocaine (4.0 min) after buccal plus lingual infiltrations. However, after articaine and lidocaine buccal infiltrations, the median onset times were identical. This difference may be the result of the higher local volume after the buccal compared to the split technique masking any differences in the two solutions that may be apparent at lower volumes. Similarly, the more rapid onset of the lidocaine buccal infiltration compared to the split technique may be a reflection of the greater buccal dose in the former method. One study ${ }^{27}$ found that the onset times of pulpal anaesthesia in lower third molars after IANBs with articaine and lidocaine were $0.93 \mathrm{~min}$ and $1.25 \mathrm{~min}$ respectively. Costa and colleagues ${ }^{28}$ reported shorter onset times in the maxillary posterior teeth when compared to the current mandibular study. This is probably because of differences in the cortical bone density and thickness between the upper and lower jaws. Oliveira and others ${ }^{29}$ found no significant differences between articaine and lidocaine in the maxillary canine region (1 min versus 3 min respectively).

Several local anaesthetic trials have used VAS to measure discomfort after local anaesthetic injections. ${ }^{2,3,7,19,22,23}$ In the present investigation, there were no significant differences in the mean pain scores between 2\% lidocaine and 4\% articaine (both with 1:100,000 adrenaline) or between the two methods of administration (buccal or buccal plus lingual). The only difference was that the lingual infiltration was more uncomfortable than lingual penetration used as the dummy injection. These outcomes are similar to those recorded in other clinical trials. ${ }^{30,31}$

\section{CONCLUSIONS}

When the number of episodes of no response to maximal pulp testing was considered as an indicator of anaesthetic efficacy, splitting the dose of local anaesthetic between buccal and lingual sides produced more episodes of anaesthesia of lower incisor teeth than a buccal dose alone. This was true for both articaine and lidocaine administration, however sustained anaesthesia did not differ between techniques.

4\% articaine with 1:100,000 adrenaline was significantly more effective than 2\% lidocaine with 1:100,000 adrenaline in obtaining sustained anaesthesia in the mandibular incisors for each method of delivery investigated.

1. Corbett I P, Ramacciato J C, Groppo F C, Meechan $J$ G. A survey of local anaesthetic use among general dental practitioners in the UK attending postgraduate courses on pain control. Br Dent J 2005; 199: 784-787.

2. Kanaa M D, Whitworth J M, Corbett I P, Meechan J G. Articaine and lidocaine mandibular buccal infiltration anesthesia: a prospective randomized double-blind cross-over study. J Endod 2006: 32: 296-298.

3. Robertson D, Nusstein J, Reader A, Beck M, McCartney M. The anesthetic efficacy of articaine in buccal infiltration of mandibular posterior teeth. J Am Dent Assoc 2007; 138: 1104-1112.

4. Abdulwahab M, Boynes S, Moore P et al. The efficacy of six local anesthetic formulations used for posterior mandibular buccal infiltration anesthesia. J Am Dent Assoc 2009; 140: 1018-1024.

5. Hinkley S A, Reader A, Beck M, Meyers W J. An evaluation of $4 \%$ prilocaine with $1: 200,000$ epinephrine and 2\% mepivacaine with 1:20,000 levonordefrin compared with $2 \%$ lidocaine with 1:100,000 epinephrine for inferior alveolar nerve block. Anesth Prog 1991; 38: 84-89.

6. Clark S, Reader A, Beck M, Meyers W J. Anesthetic efficacy of the mylohyoid nerve block and combination inferior alveolar nerve block/mylohyoid nerve block. Oral Surg Oral Med Oral Pathol Oral Radiol Endod 1999; 87: 557-563.

7. Meechan J G, Ledvinka J I. Pulpal anaesthesia for mandibular central incisor teeth: a comparison of infiltration and intraligamentary injections. Int Endod J 2002: 35: 629-634.

8. Nuzum F M, Drum M, Nusstein J, Reader A, Beck $M$. Anesthetic efficacy of articaine for combination labial plus lingual infiltrations versus labial infiltration in the mandibular lateral incisor. J Endodont 2010; 36: 952-956.

9. Certosimo A J, Archer R D. A clinical evaluation of the electric pulp tester as an indicator of local anesthesia. Oper Dent 1996; 21: 25-30.

10. McDaniel K F, Rowe N H, Charbeneau G T. Tissue response to an electric pulp tester. J Prosthet Dent 1973; 29: 84-87.

11. Dreven L J, Reader A, Beck M, Meyers W J, Weaver $J$. An evaluation of an electric pulp tester as a measure of analgesia in human vital teeth. J Endod 1987; 13: 233-238.

12. Gopikrishna V, Tinagupta $K$, Kandaswamy D. Comparison of electrical, thermal, and pulse oximetry methods for assessing pulp vitality in recently traumatized teeth. J Endod 2007; 33: 531-535.

13. Nusstein J, Reader A, Beck F M. Anesthetic efficacy of different volumes of lidocaine with epinephrine for inferior alveolar nerve blocks. Gen Dent 2002; 50: 372-375.

14. Tofoli G R, Ramacciato J C, de Oliveira P C, Volpato M C, Groppo F C, Ranali J. Comparison of effectiveness of 4\% articaine associated with 1:100,000 or $1: 200,000$ epinephrine in inferior alveolar nerve block. Anesth Prog 2003; 50: 164-168.

15. Volpato M C, Ranali J, Ramacciato J C, de Oliveira P C, Ambrosano G M, Groppo F C. Anesthetic efficacy of bupivacaine solutions in inferior alveolar nerve block. Anesth Prog 2005; 52: 132-135.

16. Branco F P, Ranali J, Ambrosano G M, Volpato M C. A double-blind comparison of $0.5 \%$ bupivacaine with 1:200,000 epinephrine and 0.5\% 
levobupivacaine with 1:200,000 epinephrine for the inferior alveolar nerve block. Oral Surg Oral Med Oral Pathol Oral Radiol Endod 2006; 101: 442-447.

17. Clark K, Reader A, Beck M, Meyers W J. Anesthetic efficacy of an infiltration in mandibular anterior teeth following an inferior alveolar nerve block. Anesth Prog 2002; 49: 49-55.

18. Vreeland D L, Reader A, Beck M, Meyers W, Weaver $J$. An evaluation of volumes and concentrations of lidocaine in human inferior alveolar nerve block. J Endod 1989; 15: 6-12.

19. Meechan J G, Kanaa M D, Corbett I P, Steen I N, Whitworth J M. Pulpal anaesthesia for mandibular permanent first molar teeth: a double-blind randomized cross-over trial comparing buccal and buccal plus lingual infiltration injections in volunteers. Int Endod J 2006; 39: 764-769.

20. Gross R, McCartney M, Reader A, Beck M. A prospective, randomized, double-blind comparison of bupivacaine and lidocaine for maxillary infiltrations. J Endod 2007: 33: 1021-1024.
21. Yonchak T, Reader A, Beck M, Clark K, Meyers W J. Anesthetic efficacy of infiltrations in mandibular anterior teeth. Anesth Prog 2001; 48: 55-60.

22. Whitworth J M, Kanaa M D, Corbett I P, Meechan $J$ G. Influence of injection speed on the effectiveness of incisive/mental nerve block: a randomized, controlled, double-blind study in adult volunteers. J Endod 2007; 33: 1149-1154.

23. Corbett I P, Kanaa M D, Whitworth J M, Meechan $J \mathrm{G}$. Articaine infiltration for anesthesia of mandibular first molars. J Endod 2008; 34: 514-518.

24. Nist R A, Reader A, Beck M, Meyers W J. An evaluation of the incisive nerve block and combination inferior alveolar and incisive nerve blocks in mandibular anesthesia. J Endod 1992; 18: 455-459.

25. Fernandez C, Reader A, Beck M, Nusstein J. A prospective, randomized, double-blind comparison of bupivacaine and lidocaine for inferior alveolar nerve blocks. J Endod 2005; 31: 499-503.

26. Haas D A, Harper D G, Saso M A, Young E R. Comparison of articaine and prilocaine anesthesia by infiltration in maxillary and mandibular arches. Anesth Prog 1990; 37: 230-237.

27. Sierra Rebolledo A, Delgado Molina E, Berini Aytis L, Gay Escoda C. Comparative study of the anesthetic efficacy of $4 \%$ articaine versus $2 \%$ lidocaine in inferior alveolar nerve block during surgical extraction of impacted lower third molars. Med Oral Patol Oral Cir Bucal 2007; 12: E139-144.

28. Costa C G, Tortamano I P, Rocha R G, Francischone

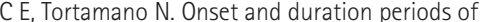
articaine and lidocaine on maxillary infiltration. Quintessence Int 2005; 36: 197-201.

29. Oliveira P C, Volpato M C, Ramacciato J C, Ranali $J$. Articaine and lignocaine efficiency in infiltration anaesthesia: a pilot study. Br Dent J 2004; 197: 45-46

30. Vahatalo $\mathrm{K}$, Antila H, Lehtinen R. Articaine and lidocaine for maxillary infiltration anesthesia. Anesth Prog 1993; 40: 114-116.

31. Malamed S F, Gagnon S, Leblanc D. Articaine hydrochloride: a study of the safety of a new amide loca anesthetic. J Am Dent Assoc 2001; 132: 177-185. 